\title{
Espumado por microondas de mezclas de silicato de sodio-ortofosfato cálcico dibásico
}

\author{
G. VARGAS, J.C. PÉREZ, J. MÉNDEZ, M. MÉNDEZ, P. PENA ${ }^{1}$ \\ CINVESTAV Unidad Saltillo, Coahuila, México. \\ 'Instituto de Cerámica y Vidrio, Madrid, España
}

\begin{abstract}
La acción de la energía de las microondas, genera porosidad interna en un material cerámico húmedo. En el presente trabajo, se analiza el efecto del tiempo de aplicación de las microondas sobre el comportamiento térmico, cambio de volumen, tamaño y distribución de poros, reacciones químicas y disolución de probetas cerámicas de silicato de sodio / ortofosfato cálcico dibásico (FCD). Para caracterizar las espumas cerámicas, se utilizó, análisis de imágenes, microscopía electrónica de barrido, difracción de rayos $\mathrm{X}$ y picnometría de helio. Se obtuvieron espumas cerámicas con una porosidad de 71 a $79 \%$ y un rango de tamaño de poro de 10 a $200 \mu \mathrm{m}$. Después de 90 segundos, las probetas alcanzaron una temperatura de aproximadamente $130^{\circ} \mathrm{C}$ y una pérdida de peso de $23 \%$ con respecto al peso total de la muestra. Se observaron cambios de volumen y de los compuestos químicos en las muestras espumadas cuando se varió la proporción del FCD en la mezcla. Para contenidos de 10, 20 y $30 \%$ en peso de FCD, el incremento de volumen se redujo a 350, 300 y $250 \%$ respectivamente. En muestras con $30 \%$ de FCD calentadas por microondas durante 90 segundos la cantidad de un nuevo compuesto en la espuma, constituido por $\beta-\mathrm{NaCaPO}_{4}$, fue del orden de $42.9 \%$. En esa misma muestra, el $57.1 \%$ restante fue FCD. Considerando pruebas de disolución de un gramo de muestra en $100 \mathrm{ml}$ de agua destilada, la disolución obtenida en muestras espumadas por microondas después de 20 días fue de 900 ppm/gr para el Na y de 2.7 ppm/gr para el Ca en muestras con $10 \%$ de FCD. En muestras con $30 \%$ de FCD la disolución de Na fue de $470 \mathrm{ppm} / \mathrm{gr}$ y del Ca de $5 \mathrm{ppm} / \mathrm{gr}$.
\end{abstract}

Palabras clave: Espumas cerámicas, microondas, silicato de sodio, fosfato de calcio.

\section{Microwave foaming of sodium silicate-ortodibasic calcium phosphate mixtures}

The effect of microwave application on the thermal behaviour, volume change, size and pore size distribution as well as chemical reactions and dissolution of sodium silicate/dibasic calcium phosphate ceramic (DCF) samples have been analysed. Ceramics foams with porosities between 71 and $79 \mathrm{wt} \%$ and pore sizes ranging from 10 to 200 microns have been obtained. After 90 seconds the temperature of samples reach about $130{ }^{\circ} \mathrm{C}$ with a weight loss of $23 \mathrm{wt} \%$. Differences in the proportion of DCF in the initial mixture originate changes in volume and different chemical compounds in the final obtained foams. Additions of 10, 20 and $30 \mathrm{wt} \%$ of DCF originates volume increases of 350, 300 and $250 \%$ respectively. In foam samples with $30 \mathrm{wt} \%$ of DCF, heated by microwave during $90 \mathrm{sec}$, the amount of the new compound $\beta-\mathrm{NaCaPO}_{4}$ was about $42.9 \mathrm{wt} \%$. The other component of this sample, $57.1 \mathrm{wt} \%$, was DCF. Dissolution tests were made, using one gram of sample, in $100 \mathrm{ml}$ of distilled water. The dissolution of $10 \mathrm{wt} \%$ DCF foamed samples, after 20 days into water, were $900 \mathrm{ppm} / \mathrm{gr}$ of Na and 2.7 $\mathrm{ppm} / \mathrm{gr}$ of Ca.

In samples with $30 \mathrm{wt} \%$ of DCF the dissolution were $470 \mathrm{ppm} / \mathrm{gr}$ and $5 \mathrm{ppm} / \mathrm{gr}$ for Na and Ca respectively.

Key words: ceramic foams, microwaves, sodium silicate, calcium phosphate

\section{1. - INTRODUCCIÓN}

Por su estructura, existen básicamente dos categorías de espumas cerámicas. Las de poro abierto, utilizadas en aplicaciones que involucran transporte de un fluido como filtración y catálisis, y las espumas de poro cerrado; las cuales tienen aplicaciones como aislantes térmicos (1). Para producir piezas cerámicas de porosidad controlada, se han desarrollado varios métodos (1-7): gelación de soluciones, gelación de microemulsiones, tratamiento hidrotermal, uso de aditivos espumantes y espumado térmico. La elección de algún método específico depende entre otras cosas, de la aplicación o función para la cual se diseña el producto y de su costo. Un método sencillo y de bajo costo para la fabricación de espumas cerámicas de porosidad cerrada es el espumado térmico. El espumado térmico de un material cerámico puede realizarse mediante un calentamiento convencio- nal (radiación - convección) o por microondas. Al calentar una pieza por métodos convencionales, el gradiente de temperatura y el gradiente de humedad tienen direcciones opuestas, el efecto neto resultante es usualmente la formación de una capa superficial densa y casi libre de poros abiertos que dificulta la transmisión de calor del exterior hacia el interior del cuerpo cerámico. El producto resultante son piezas con porosidad macroscópica difícil de controlar. En el proceso de calentamiento por microondas, el calor es generado internamente en el material. Como resultado de este calentamiento, los gradientes térmicos y el flujo de calor en materiales procesados por microondas son el inverso de aquellos procesados por métodos convencionales, creando una distribución de temperatura con un máximo en el centro de la muestra calentada $(8,9)$. 
Las microondas obedecen las leyes de la óptica por lo que pueden ser transmitidas, absorbidas, y reflejadas, dependiendo del tipo de material. Los metales son opacos a las microondas. Los materiales cerámicos dieléctricos $\left(\mathrm{Al}_{2} \mathrm{O}_{3^{\prime}}\right.$, $\mathrm{MgO}, \mathrm{SiO}_{2}$ y la mayoría de los vidrios), son transparentes a las microondas a temperatura ambiente (9). El calentamiento por microondas de un material, es el resultado de la absorción de energía de microondas por el material expuesto a un campo electromagnético en el rango de frecuencias de 300 $\mathrm{MHz}$ a $300 \mathrm{GHz}$ (8). A medida que la temperatura de estos materiales se incrementa, su capacidad de absorción de las microondas aumenta considerablemente. La temperatura crítica $\left(\mathrm{T}_{\text {crit }}\right)$ es la temperatura a la cual los materiales cerámicos comienzan a absorber y a calentarse más eficientemente mediante radiación de microondas. Por ejemplo para la alúmina la $\mathrm{T}_{\text {crit }}$ es de $600{ }^{\circ} \mathrm{C}$. La absorción de las microondas por cerámicos transparentes puede ser enormemente aumentada por la acción de algunos aditivos o por la presencia de humedad. Meek (10), reportó la fabricación de espumas inorgánicas a partir de: micro esferas de vidrio, cenizas volantes, microesferas metálicas y fibras de vidrio. En este trabajo, se utilizaron silicatos alcalinos como materiales absorbentes de las microondas a los que se les llama agentes de acoplamiento. Cuando se preparan mezclas de materiales cerámicos con silicatos alcalinos, en la superficie de las piezas consolidadas, se forma una capa densa que al secarse evita la evaporación total de la humedad en la pieza cerámica. El calentamiento en dichas condiciones, dificulta la eliminación de la humedad interna, espumándose la muestra de acuerdo a la rapidez del calentamiento.

De acuerdo a Braykov (11), el proceso de secado de soluciones de silicatos alcalinos en una cavidad de microondas puede ser dividido en cuatro etapas:

Calentamiento de la solución hasta la temperatura de ebullición (sin cambio importante en el contenido de agua).

Evaporación del agua libre a una velocidad constante (bajo incremento de la temperatura).

Desaceleración del secado (acompañado por un notable incremento de la temperatura); y eliminación de la humedad residual (elevación de la temperatura hasta $120-190{ }^{\circ} \mathrm{C}$ dependiendo de la relación molar $\mathrm{SiO}_{2} / \mathrm{M}_{2} \mathrm{O}$ ).

En el presente trabajo, se analiza el efecto del tiempo de aplicación de microondas sobre el comportamiento térmico, cambio de volumen, tamaño y distribución de poros, reacciones químicas y disolución de probetas cerámicas de silicato de sodio / ortofosfato cálcico dibásico (FCD); con el propósito de desarrollar un procedimiento para obtener piezas cerámicas de porosidad controlada. Como el silicato de sodio es un compuesto muy soluble en soluciones acuosas, la incorporación del FCD se realizó para evaluar si en tiempos cortos es posible formar productos de reacción menos solubles y por otra parte analizar si su incorporación al silicato de sodio afecta el grado de espumado y la distribución del tamaño de los poros resultantes en las piezas cerámicas.

\section{PROCEDIMIENTO EXPERIMENTAL.}

Materiales utilizados. El tamaño de las partículas de silicato de sodio utilizado fue de 6 a $40 \mu \mathrm{m}$. Su composición química fue la siguiente: $61,8 \%$ de $\mathrm{SiO}_{2}, 19,2 \%$ de $\mathrm{Na}_{2} \mathrm{O}$ y $18,5 \%$ de $\mathrm{H}_{2} \mathrm{O}$. El Fosfato cálcico dibásico grado reactivo, suministrado por Aldrich, fue usado en un rango de tamaño de partícula similar el del silicato de sodio. Para humedecer las mez- clas de silicato de sodio- FCD, se utilizó agua destilada y desionizada.

Preparación de probetas. La preparación de las probetas fue realizada de la manera siguiente:

Se prepararon mezclas de silicato de sodio con 10, 20 y 30 $\%$ en peso de fosfato cálcico dibásico. Las mezclas fueron homogenizadas en un molino planetario, durante 15 minutos.

Se pesaron 2 gr de mezcla y se colocaron en una matriz cuadrada de acero inoxidable, a la cual posteriormente se le agregaron 0,5 $\mathrm{ml}$ de agua justo antes de aplicar una presión de 400MPa durante 15 segundos. Posteriormente, se desmontó la probeta.

Se colocaron las muestras en una estufa a $30^{\circ} \mathrm{C}$ durante 4 horas. Posteriormente se pesaron, se espumaron térmicamente y se pesaron nuevamente para registrar su pérdida de peso.

Equipo experimental. Las pruebas de espumado por microondas se realizaron en una cavidad multimodo CEM modelo MARS 5. En todas las pruebas se trabajó a una potencia de 1200 watts y a una frecuencia de $2450 \mathrm{MHz}$. Este horno cuenta con un sistema para medición de temperatura a base de un sensor de fibra óptica, que permite realizar mediciones hasta $300{ }^{\circ} \mathrm{C}$.

Técnicas de caracterización. Para obtener imágenes con humedad presente en las muestras, se utilizó un microscopio electrónico de barrido Philips modelo XL30 en modo ambiental. Las muestras fueron cortadas transversalmente para obtener imágenes de la porosidad interna de las muestras espumadas. La medición de la distribución y tamaño de poros fue realizada mediante el análisis de imágenes a 2 micrografías del centro y orilla de cada una de las muestras espumadas sometidas a fractura transversal. Se utilizó el comando de medición / conteos en la modalidad de medición de diámetro promedio, el cual obtiene el promedio de tres ejes ( $a$, b y c) trazados en ángulos de $120^{\circ}$, pasando por el centro del poro. La estructura cristalina fue caracterizada mediante un difractómetro de rayos $X$ marca Philips, operado con radiación de $\mathrm{Cu}$ $(\lambda=1,542 \AA)$, a una velocidad de barrido de $0,02(2 \theta / \mathrm{seg})$ y un voltaje de excitación del ánodo de $40 \mathrm{KV}$. La cuantificación de fases se obtuvo por el Método Rietveld (12). Las mediciones del volumen de las muestras espumadas se realizaron mediante el principio de Arquímedes. La densidad real de los polvos cerámicos fue determinada mediante la técnica de picnometría de gas (He/Aire) en un multipicnómetro operado a una presión del helio de 2,07MPa. Para medir la densidad aparente se utilizó el principio de Arquímedes usando mercurio en vez de agua como medio para evitar que el fluido penetrara en los poros. La porosidad de las muestras fue calculada considerando la siguiente ecuación.

$$
\text { Porosidad }=\left[1-\left[\frac{\text { volumen de solido }}{\text { volumen de solido }+ \text { volumen de poros }}\right]\right]=\left[1-\left(\frac{\rho_{\text {aparente }}}{\rho_{\text {real }}}\right)\right]
$$

Las pruebas de solubilidad se realizaron a temperatura ambiente durante 20 días, las muestras espumadas, fueron molidas y pasadas por malla 100; 1 gramo de polvo se colocó en frascos de plástico conteniendo $100 \mathrm{ml}$ de agua desionizada a temperatura ambiente. Los resultados de solubilidad se obtuvieron mediante la cuantificación del cambio de concentración de Ca y Na de la solución obtenida después de filtrar el polvo del agua contenida en el frasco, las mediciones se realizaron mediante absorción atómica. 


\section{3.- RESULTADOS}

Comportamiento de la temperatura y de la pérdida de peso. Con el propósito de evaluar los patrones de calentamiento de las diferentes mezclas de materiales, se realizaron mediciones de temperatura durante el espumado térmico por microondas. Las muestras fueron introducidas a una temperatura de $30 \pm 3{ }^{\circ} \mathrm{C}$ en el horno de microondas. Las mediciones de temperatura se realizaron cada segundo durante 90 segundos. La figura 1 muestra la evolución del cambio de temperatura de las tres mezclas estudiadas. En esta figura, se pueden distinguir básicamente tres etapas en el calentamiento por microondas; la primer zona corresponde a un calentamiento rápido en la cual se alcanzaron temperaturas en el centro de la muestra entre 80 y $90^{\circ} \mathrm{C}$; la segunda en donde se presentó una rapidez de calentamiento más moderada; y la tercera en la que se vuelve a incrementar rápidamente la temperatura. En la última etapa se alcanzó una temperatura de $130^{\circ} \mathrm{C}$. Es importante indicar que el tiempo de tratamiento fue limitado a 90 segundos para proteger la cámara del horno debido a que en ensayos preliminares en que se prolongó el tiempo de tratamiento se llegaron a fundir las muestras rápidamente. En esta gráfica también se puede observar que las adiciones de FCD no ocasionaron cambios importantes en los perfiles térmicos de las probetas. En la figura 2 podemos observar que durante los primeros 20 segundos, se alcanza una pérdida de peso aproximada del $13 \%$, dicho lapso de tiempo es suficiente para calentar y evaporar el agua libre, fluyendo vapor hacia la superficie de la muestra, esto debido al calentamiento volumétrico (13). En la segunda etapa, en la que se presenta una rapidez de pérdida de peso más moderada, se elimina el agua químicamente ligada, continuando el flujo de agua caliente y de vapor del centro hacia la superficie de la muestra. El agua químicamente ligada es más difícil de eliminar debido a la menor movilidad de las moléculas ante la acción del campo eléctrico creado por las microondas (13). Después de 90 segundos de calentamiento, se alcanzó una pérdida de peso entre el 21 y el $23 \%$.

En la figura 3, se compara la pérdida de peso de una muestra de silicato de sodio con $30 \%$ de FCD, tanto en calentamiento convencional como en calentamiento mediante microondas. Mientras en el calentamiento convencional se alcanzó una pérdida de peso del orden del 10\% después de 20 minutos de calentamiento a $130{ }^{\circ} \mathrm{C}$, en el secado por microondas se alcanzó una pérdida de peso del $23 \%$ en 1 y 5 minutos. En el calentamiento convencional, primero se calienta la superficie, posteriormente el calor debe difundir hacia el centro de la pieza y calentar el agua, lo que hace que fluya hacia la superficie, donde es eliminada. La etapa de secado del centro es limitada por la conductividad térmica del material; debido a esto el secado de la mayoría de materiales cerámicos por el método convencional es muy lento (14).

Cambio de volumen de muestras espumadas en microondas. El calor durante el secado, genera presión interna en toda la pieza, debido a la evaporación local, generando esfuerzos mecánicos que pueden llegar a la ruptura. La figura 4, muestra el cambio de volumen en función del tiempo para diferentes muestras espumadas mediante microondas. El cambio de volumen se debe a la presión interna generada por el flujo de agua caliente y vapor provocado por el calentamiento volumétrico de las microondas.

Los cambios de volumen de las muestras con $30 \%$ y $10 \%$ de FCD después de 90 segundos fueron de 250 a $350 \%$ respectivamente. Esta variación implica que un cambio en la adición de $10 \%$ a $30 \%$ de FCD al silicato de sodio reduce el cambio volumétrico en un $28,5 \%$.

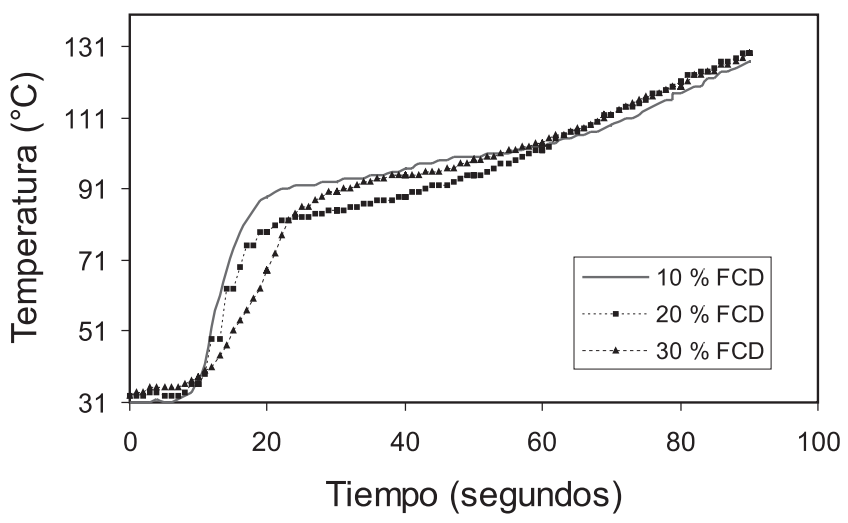

Figura 1. Evolución térmica de muestras de silicato de sodio con 10, 20 y $30 \%$ de FCD durante el espumado térmico por microondas.

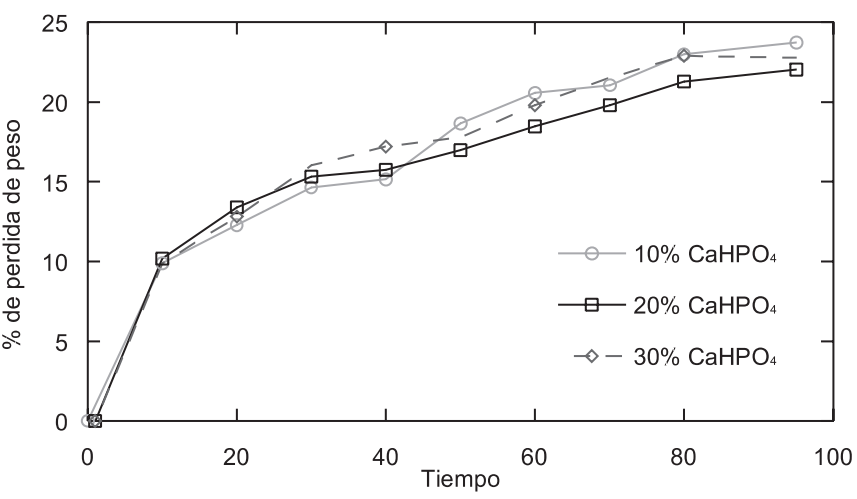

Figura 2. Evolución de la pérdida de peso de muestras de silicato de sodio con 10, 20 y 30\% de FCD durante el espumado térmico por microondas. (tiempo en segundos)

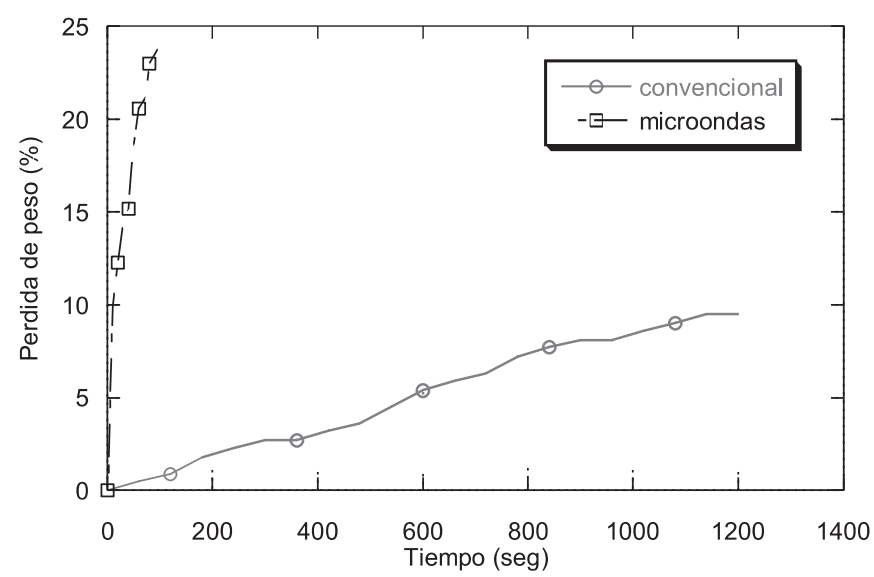

Figura 3. Pérdida de peso de muestras de silicato de sodio con $30 \%$ de FCD, calentadas mediante microondas y convencionalmente a $130{ }^{\circ} \mathrm{C}$. 
Tamaño y distribución de poros de muestras espumadas por microondas. En la figura 5, se muestra una fotomicrografía de una probeta de $\mathrm{Na}_{2} \mathrm{SiO}_{4}$ con $20 \%$ de $\mathrm{CaHPO}_{4}$ espumada mediante microondas durante 90 segundos. En esta figura, es posible observar tamaños de poro entre 20 a $80 \mu \mathrm{m}$ con una matriz de paredes muy delgadas que confina los poros.

La figura 6, muestra la distribución del tamaño de poros de las muestras espumadas en microondas durante 90 segundos de muestras de silicato de sodio con 10, 20 y 30\% de FCD. Como se puede observar, las muestras con $10 \%$ de FCD, presentan una distribución de tamaño de poros más amplia $(10$ a $200 \mu \mathrm{m})$ con un tamaño medio de $100 \mu \mathrm{m}$. Las muestras con $20 \%$ de FCD presentaron poros en el rango de 10 a $150 \mu \mathrm{m}$ con un tamaño medio de $60 \mu \mathrm{m}$. La muestra con $30 \%$ de FCD presentó una gran cantidad de poros en el rango de 10 a $60 \mu \mathrm{m}$ de un tamaño promedio de $30 \mu \mathrm{m}$. Analizando las curvas es evidente que a mayor cantidad de FCD el tamaño de poros se redujo.

Porosidad de muestras espumadas mediante microondas. La tabla 1, presenta los valores de porosidad obtenidos en espumas de mezclas de silicato de sodio con 10,20 y $30 \%$ en peso de $\mathrm{CaHPO}_{4}$ expuestas a la energía de microondas durante 70, 80 y 90 segundos.

En la tabla 1, podemos observar que en muestras con $10 \%$ de FCD la porosidad disminuyó de 79 a $75 \%$ cuando el tiempo de exposición a las microondas pasó de 70 a 80 segundos. De 80 a 90 segundos no se observó cambio de la porosidad por lo que se puede inferir que 75 segundos de exposición son suficientes para alcanzar la máxima porosidad durante el espumado por microondas. Por otra parte, para un tiempo de exposición de 90 segundos, la porosidad de las muestras con $10 \%$ de FCD ( $75 \%$ ) es muy similar a la de las muestras con 20 y $30 \%$ de FCD (71 y $73 \%$ respectivamente) considerando la desviación estándar de los resultados $( \pm 2 \%)$. Esto significa que a pesar de haberse obtenido una reducción en el cambio de volumen de las muestras con mayor cantidad de FCD, estas presentaron una distribución de poros más pequeños, lo cual podría explicar porqué la porosidad resultó muy similar. Estos resultados no permiten determinar la relación de porosidad abierta a porosidad cerrada, sin embargo es de esperar que al ser eliminada el agua de la muestra exista porosidad abierta hasta la superficie. Esto se puede corroborar si observamos los poros de la superficie de la muestra espumada mediante microondas (figura 7). La porosidad superficial presentada en la micrografía de la figura 7 , muestra la formación de una densidad superficial de poros aproximada de 50poros $/ \mathrm{mm}^{2}$, con un tamaño promedio de $15 \mu \mathrm{m}$.

TABla I Porosidad (\%) DE MUESTRAS ESPUMADAS MEDIANTE MICROONDAS, $(x=10 \%, y=20 \%$ y $z=30 \%$ de FCD RESPECTIVAMENTE); ( $\mathrm{a}=70, \mathrm{~b}=80 \mathrm{y} \mathrm{c}=90$ SEGUNDOS DE EXPOSICIÓN $)$

\begin{tabular}{|c|c|c|c|}
\hline Muestra & $\begin{array}{c}\text { Densidad real } \\
\left(\mathbf{g r} / \mathbf{c m}^{\mathbf{3}}\right)\end{array}$ & $\begin{array}{c}\text { Densidad aparente } \\
\left(\mathbf{g r} / \mathbf{c m}^{\mathbf{3}}\right)\end{array}$ & $\begin{array}{c}\text { Porosidad } \\
\mathbf{( \% )}\end{array}$ \\
\hline $10 \%$ FCD; 70seg. & 1.678 & $0.36 \pm 0.02$ & $79 \pm 2$ \\
\hline $10 \%$ FCD; 80seg & 1.232 & $0.31 \pm 0.03$ & $75 \pm 2$ \\
\hline $10 \%$ FCD; 90seg & 1.3019 & $0.32 \pm 0.02$ & $75 \pm 2$ \\
\hline $20 \%$ FCD; 90seg & 1.3069 & $0.376 \pm 0.01$ & $71 \pm 2$ \\
\hline $30 \%$ FCD; 90seg & 1.7445 & $0.46 \pm 0.04$ & $73 \pm 2$ \\
\hline
\end{tabular}

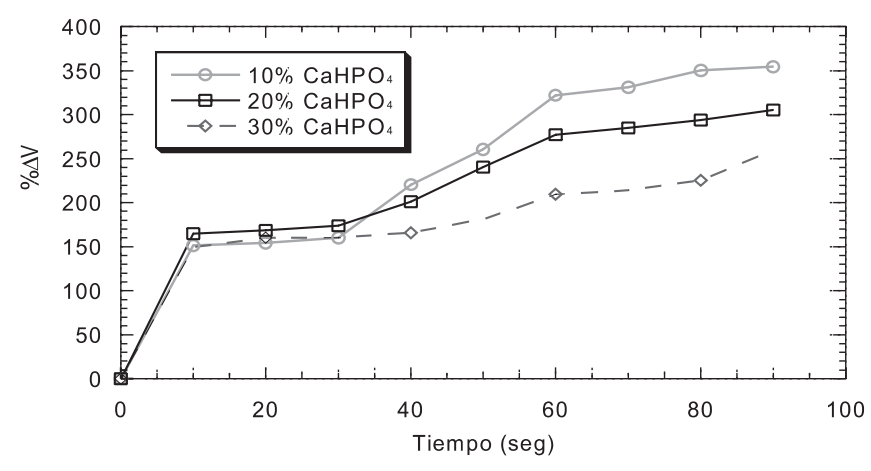

Figura 4. Cambio de volumen (\%) de muestras espumadas mediante microondas

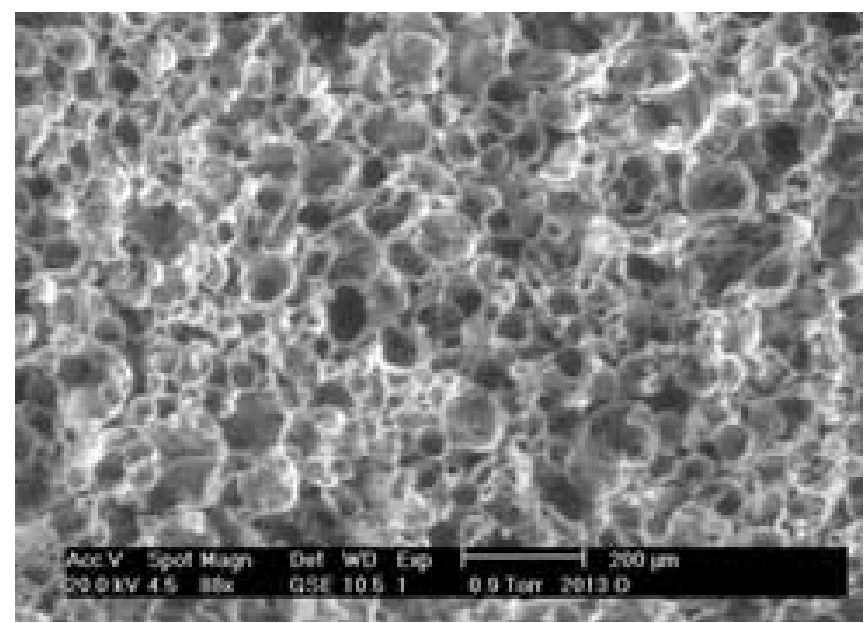

Figura 5. Muestra de silicato de sodio con $20 \%$ de $\mathrm{CaHPO}_{4}$ espumada por microondas

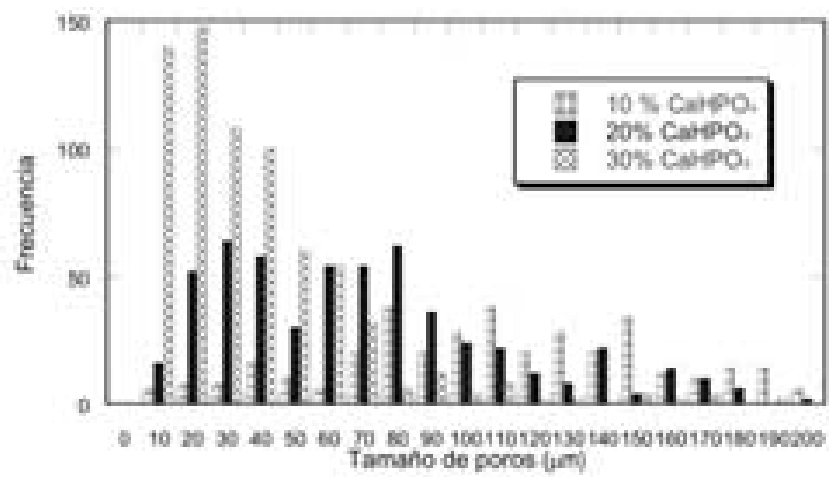

Figura 6. Distribución del tamaño de poros de muestras espumadas mediante microondas. 
Efecto del calentamiento por microondas sobre la composición química de las espumas cerámicas. La figura 8 , muestra los difractogramas de muestras de silicato de sodio con $30 \%$ de FCD sometidas a la acción de las microondas durante 70 y 90 segundos. En estos difractogramas aparecen señales características del FCD, y de una nueva fase constituida por fosfato cálcico de sodio $\left(\beta-\mathrm{NaCaPO}_{4}\right)$ junto con una ancha banda entre 20 y $352 \theta$ que se puede atribuir a la presencia de una fase amorfa. La presencia del $\beta-\mathrm{NaCaPO}_{4}$, indica que durante el procesamiento por microondas no solamente la eliminación de agua es rápida, sino que también se pueden producir reacciones químicas en tiempos relativamente cortos, lo cual ha sido previamente reportado para otros sistemas (15). Los resultados cuantitativos mediante el método Rietveld (12), mostraron que en una muestra con $30 \%$ de FCD espumada durante 70 segundos mediante microondas, la relación $\beta-\mathrm{NaCaPO}_{4} / \mathrm{CaPO}_{3}(\mathrm{OH})$ es del orden de 22,9\%/77,1\%. Considerando que las microondas pueden favorecer la reacción de formación del $\beta-\mathrm{NaCaPO}_{4}$, a mayor tiempo de exposición, mayor será la cantidad de dicha fase, por lo que se realizó una cuantificación de fases a una muestra de la misma composición que la anterior, pero esta vez espumada mediante microondas durante 90 segundos. Los resultados de la cuantificación mostraron que la cantidad de $\beta-\mathrm{NaCaPO}_{4}$ fue del orden de $42,9 \%$ mientras que el contenido de $\mathrm{CaPO}_{3}(\mathrm{OH})$ fue de $57,1 \%$. Considerando estos valores podemos decir que la fase $\beta-\mathrm{NaCaPO}_{4}$ aumentó $20 \%$ en solo 20 segundos bajo el campo de acción de las microondas.

Solubilidad de espumas producidas mediante microondas. La tabla 2 muestra los resultados de solubilidad obtenidos después de 20 días de muestras de silicato de sodio con 10, 20 y 30\% de FCD espumadas mediante microondas. En todos los casos la solubilidad fue evaluada considerando un gramo de muestra en $100 \mathrm{ml}$ de agua destilada.

Tal como se puede observar en la tabla 2, el elemento más soluble es el sodio. Esta solubilidad disminuyó a medida que se incrementó la adición del FCD. De esto se puede inferir que a menor contenido de silicato de sodio sin reaccionar, menor será la solubilidad del sodio debido a que el silicato de sodio es más soluble que el FCD y que la nueva fase $\mathrm{NaCaPO}_{4}$. La disolución obtenida en muestras espumadas por microondas después de 20 días en agua destilada fue de 900 ppm/gr para el Na y de 2,7 ppm/gr para el Ca para muestras con $10 \%$ de FCD. En muestras con 30\% de FCD la disolución de Na fue de $470 \mathrm{ppm} / \mathrm{gr}$ y del Ca de $5 \mathrm{ppm} / \mathrm{gr}$. Así pues la extracción de calcio esta en proporción directa con el contenido de este elemento en la muestra, mientras que la de sodio se reduce en mucha mayor medida con el contenido en silicato sódico.

\section{CONCLUSIONES.}

Durante el calentamiento por microondas de mezclas de silicato de sodio-FCD se alcanza una temperatura de $130^{\circ} \mathrm{C}$, en un tiempo de 90 segundos. En este periodo, se produce una pérdida de peso de alrededor del $23 \%$ del peso total de la muestra.

La adición de FCD, disminuye el cambio de volumen de las muestras, lo que indica que el silicato de sodio es el material que favorece el espumado. Sin embargo, a medida que se incrementa la proporción de FCD, se favorece la generación de poros más pequeños.

La mezcla de materiales que forman la espuma producida por microondas, reaccionan obteniendo, a 90 segundos, $42,9 \%$

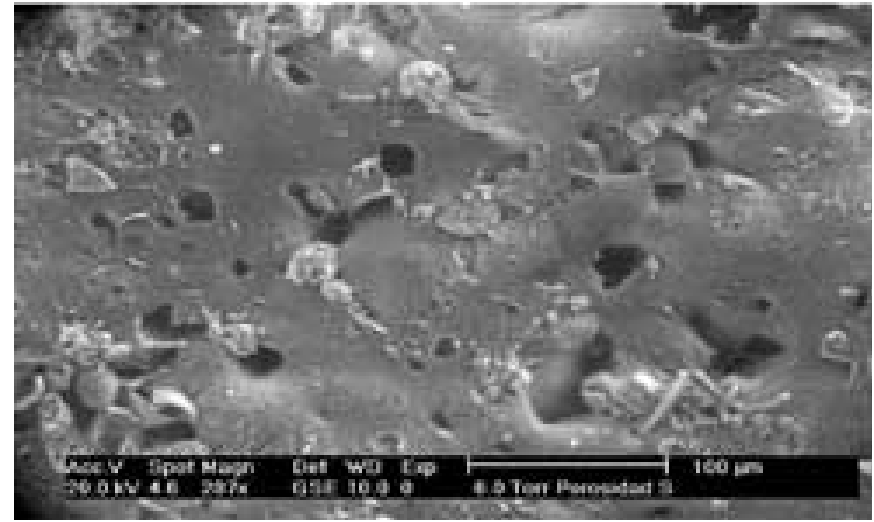

Figura 7 Porosidad superficial de una muestra producida mediante microondas.

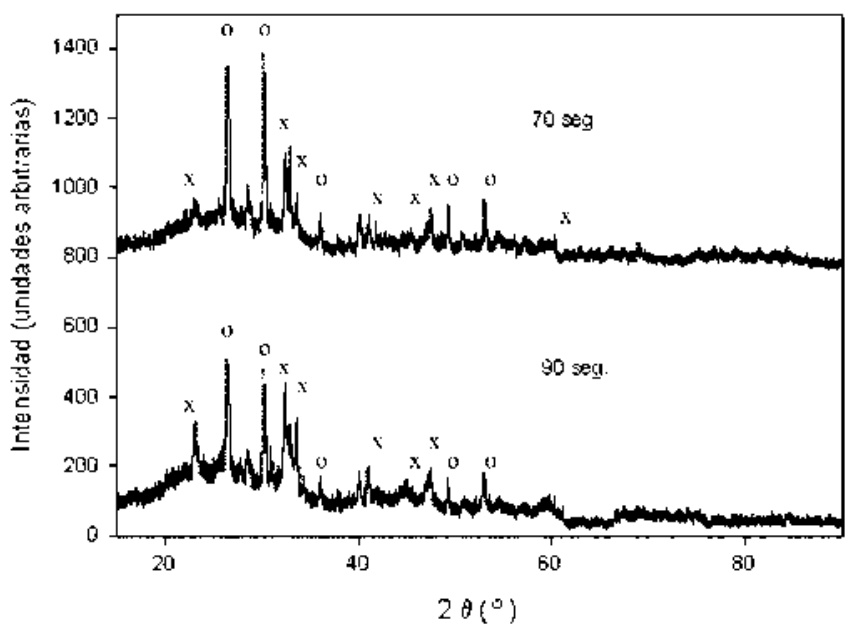

Figura 8. Difractogramas de muestras de silicato de sodio con $30 \%$ en peso de FCD, espumadas mediante microondas durante 70 y 90 segundos. $\mathrm{o}\left(\mathrm{CaPO}_{3} \mathrm{OH}\right), \mathrm{x}\left(\mathrm{NaCaPO}_{4}\right)$

TABla 2 SOlubilidad DURANTE 20 díAs A temperatura AMBIENTE, DE MUESTRAS ESPUMADAS MEDIANTE MICROONDAS

\begin{tabular}{|c|c|c|}
\hline Muestra & $\begin{array}{c}\mathrm{Ca} \\
(\mathrm{ppm}) / \mathrm{gr} \\
\text { en } 100 \mathrm{ml} \text { de agua }\end{array}$ & $\begin{array}{c}\mathrm{Na} \\
(\mathrm{ppm}) / \mathrm{gr} \\
\text { en } 100 \mathrm{ml} \text { de agua }\end{array}$ \\
\hline $10 \% \mathrm{FCD}$ & 2.7 & 901.6 \\
\hline $20 \% \mathrm{FCD}$ & 4.08 & 648.8 \\
\hline $30 \% \mathrm{FCD}$ & 5.82 & 471.8 \\
\hline
\end{tabular}


de $\mathrm{NaCaPO}_{4}$ con respecto al total de la fase cristalina de la muestra ( $\mathrm{FCD}_{4} \mathrm{NaCaPO}_{4}$ ).

La porosidad de las muestras de silicato de sodio-FCD, espumadas durante 90 segundos, mediante microondas, se encuentra en el rango de 71 a $75 \pm 2 \%$.

El tamaño y distribución de poros de las muestras de silicato de sodio-FCD, espumadas mediante microondas durante 90 segundos, se encuentra en el rango de 60 a $160 \mu \mathrm{m}$ para muestras con $10 \%$ de FCD, de 20 a $100 \mu$ m para muestras con $20 \%$ de FCD y de 10 a $60 \mu$ m para muestras con $30 \%$ de FCD

Considerando pruebas de disolución de un gramo de muestra en $100 \mathrm{ml}$ de agua destilada, la disolución obtenida en muestras espumadas por microondas después de 20 días fue de $900 \mathrm{ppm} / \mathrm{gr}$ apara el Na y de 2,7 ppm/gr para el Ca en muestras con 10\% de FCD. En muestras con 30\% de FCD la disolución de Na fue de 470 ppm/gr y del Ca de 5 ppm/gr.

\section{BIBLIOGRAFÍA}

1. W. P. Minnear, (1992), "Processing of Porous Ceramic", American Ceramic Society Bulletin, Vol. 71, No. 11, pp. 1674-82

2. J. Rouquerol, D. Avnir, C. W. Fairbridge, D. H. Everett, J. H. Haynes, N. Pernicone, J.D. F. Ramsay, K. S. W. Sing y K. K. Unger; (1994) "Recommendations for the Characterization of porous solids"; Pure and appl. Chem., Vol. 66, [8], pp. 1739-1758.

3. H. Motoki, "Process for Preparing a Foamed Body," U. S. Pat. No. 4084 980, Apr. 18, 1978
4. R. D. Shoup, (1976), "Controlled pore silica bodies gelled form silica solalkali silicate mixtures," in Colloid and Interface Science, Vol. 3, Academic Press, New York, pp. 63-69.

5. G. Jackson and W. Meredith, "Inorganic Foams," U. S. Pat. 4547 469, Oct. 1985.

6. R. L. Helferich and R. C. Schenck, "Processing for Producing Porous Ceramic Filter for Filtering of Particulates from Diesel Engine Exhaust Gases", U. S. Pat. No. 4871 485, Oct. 3, 1989.

7. H. Nikajima, T. Ito and Y. Muragachi, "Alumina Porous Body and Production of the Same," U.S. Pat. No. 4965 230, Oct. 23, 1990.

8. D. E. Clark, D. C. Folz and J. K. West, (2000), "Processing materials with microwave energy," Materials Science and Engineering A287, pp. 153-158.

9. W. H. Sutton, (1989), "Microwave Processing of Ceramic Materials", American Ceramic Society Bulletin, Vol. 71, No. 11, pp. 376-386.

10. T. Meek, R. Blake and T. Gregory, (1985), "Low-Density Inorganic Foams Fabricated Using Microwaves," Ceram. Eng. Sci. Proc., Vol. 6 [7-8], pp. 1161-70.

11. Brykov y L. Rikenglaz, (2000), "Simplified theory of microwave drying of alkali metal silicate solutions with arbitrary values of $\mathrm{SiO}_{2} / \mathrm{M}_{2} \mathrm{O}$ mole ratio," Journal of Microwave Power and Electromagnetic Energy, Vol. 35[3], pp. 191-196.

12. D. B. Wiles y R. A Young, A new computer program for Rietveld análisis of X - ray powder diffraction patterns, J. Appl. Cryst. (1981). 14, 149-151.

13. R. Meredith, (1998), Engineers' Handbook of Industrial Microwave Heating, IEEE Power 25, ed. The Institution of Electrical Engineers, London.

14. J. Thuery, (1992), "Microwaves: Industrial, Scientific and Medical Applications", Artech House Inc.

15. H. M. Kingston y L. B. Jassie, (1988), Introduction to Microwave Sample Preparation, ACS Professional Reference Book. Characterization of porous solids"; Pure and appl. Chem., Vol. 66, [8], pp. 1739-1758.

Recibido:

Aceptado: 